\title{
Epidermoid Cyst with Foreign Body Granuloma Formation in Hip: A Case Misdiagnosed by Ultrasound
}

\author{
Shanyong Mao, MD ${ }^{a}$, Yining Xiang, MD ${ }^{b}$, Haiyan Zhou, MD ${ }^{c}$, Linkun Zhang, MD ${ }^{a}$, Tingting Chen, MD ${ }^{a}$, \\ Bei Zhang, MD ${ }^{a, *}$ \\ ${ }^{a}$ Department of Ultrasound Medicine, The Affiliated Hospital of Guizhou Medical University, Guiyang, China; ${ }^{b}$ Department of \\ Pathology, The Affiliated Hospital of Guizhou Medical University, Guiyang, China; ${ }^{c}$ Department of Clinical Research Centre, The \\ Affiliated Hospital of Guizhou Medical University, Guiyang, China
}

Received January 18, 2020; revision received February 26, 2020; accepted February 28, 2020

\begin{abstract}
Epidermoid cysts are common benign lesions of the skin. High-frequency ultrasound is a good method for evaluation of skin soft-tissue mass. Here, we reported a case of epidermoid cyst which was misdiagnosed by ultrasound imaging as a dermoid cyst. With retrospective analysis of the ultrasound imaging, we realized that edidermoid cysts may have following features, in which the lesion originated from the skin, and have expansive hair follicle-like structure at the skin junction, as well as have onion-like or mixed echogenic changes. Ultrasound imaging can improve diagnostic accuracy through these characteristics along with clinical information.
\end{abstract}

Key words: Hip; Epidermoid; Ultrasound; Foreign body granuloma

Advanced Ultrasound in Diagnosis and Therapy 2020;04:349-351

DOI: $10.37015 /$ AUDT.2020.200004

$\mathrm{E}$ pidermoid cyst, known as Keratinocyte, usually can be found in the superficial layer of the skin, subcutaneous tissue of the head, face, neck and trunk, which are mostly single-compartment and clinically asymptomatic [1]. It is often caused by the damage of hair follicle sebaceous gland. The wall of the cyst is mostly composed by epidermal cells accompanying with granular cell layer. The cavity of epidermoid cyst contains Lamellar keratin. This type of cyst is mostly derived from the hair follicle funnel of the skin(non-implantable damage) [2]. Some scholars hold the view about the formation of Epidermoid cysts [3]. After skin injured, when foreign matter transplanted into the subcutaneous, it will stimulate tissue and grow up gradually, finally forming a walled cavity to become a cyst (implantable damage). Although epidermoid cyst is called as a cyst, it is easy to be confused with skin lesions such as dermoid cysts and sebaceous adenomas by ultrasound imaging. We report a case of epidermoid cyst with foreign body granuloma in hip, which was misdiagnosed by ultrasound examination before surgery.

\section{Case report}

The patient is a 29 -year-old male, who occasionally found a small peanut-sized mass on his left hip over 20 years ago. The mass was soft, movable, no obvious pain,and with clear boundary. Because of no clinical symptoms at the beginning,patient did not look for medical treatment. Although the mass increases the size over the years, it has no obvious painful until a week ago. When mass was squeezed by hand, patient complained pain. The size of the mass enlarged with measurement of $60 \times 30 \mathrm{~mm}$.

\footnotetext{
* Corresponding Author: Department of Ultrasound Medicine, The Affiliated Hospital of Guizhou Medical University, No. 28, Guiyi Street, Guiyang, Guizhou 550004, China.

e-mail: zhangbeixfys@163.com unrestricted use, distribution and reproduction in any medium provided that the original work is properly attributed.
} 


\section{Ultrasound examination}

Gray-scale ultrasound imaging of the mass demonstrated a solid hypo- to hyper-echoic lesion located at subcutaneous fat layers of the skin on the medial side of the left hip. The measurement of the mass was $60 \times 20 \mathrm{~mm}$ in size. The boundary of mass was clear and close to the skin layer while the internal echogenicity was heterogeneous. Color Doppler Flow Imaging shown minimal blood flow signals within the mass (Fig. 1). The ultrasound impression was a mixed echoic mass in subcutaneous tissue on the left hip, which may originate from the skin and considered as a dermoid cyst.
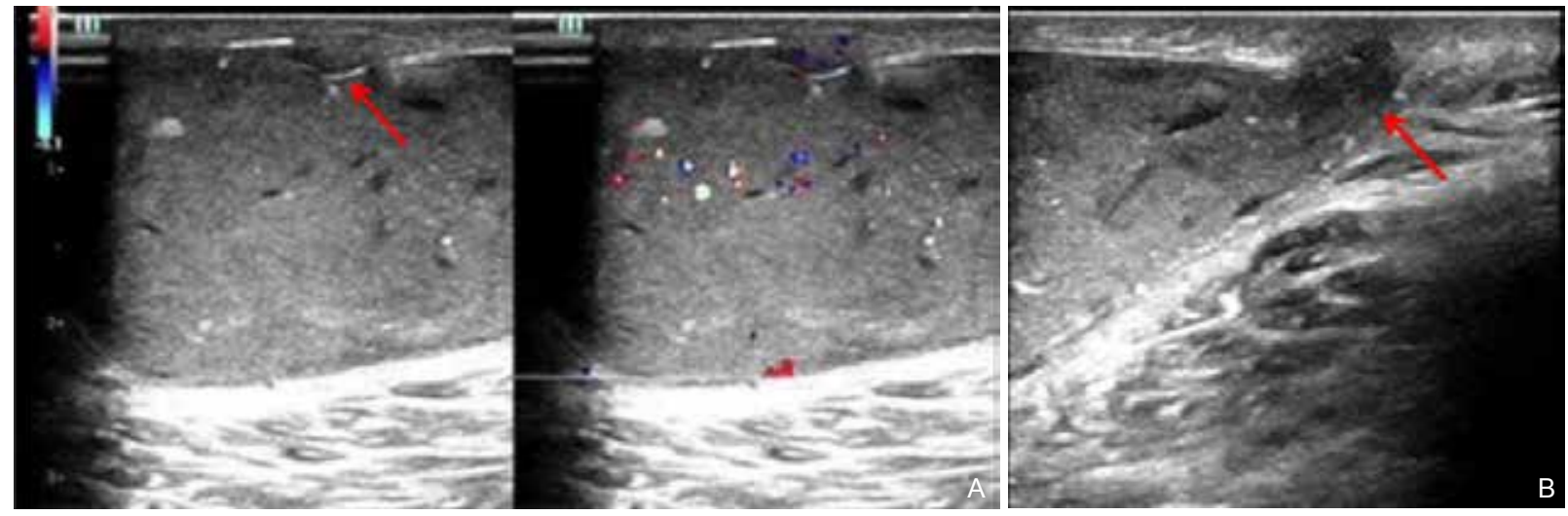

Figure 1 (A) Gray-scale and color Doppler ultrasound imaging demonstrated that the mass was hypo- and hyper-echoic lesion with clear boundary. The internal echo of the lesion was uneven and the blood flow signal of the mass interior is minimal. The mass is closely located to the skin layer (arrow); (B) The mass is closely located to the skin layer (arrow).

\section{Surgery and pathology}

Patient underwent surgery to remove the mass. A dark yellow mass measuring $50 \times 40 \times 30 \mathrm{~mm}$ in the subcutaneous tissue was removed. The lesion was soft with complete capsule. There was no obvious lesion tissue in the margin and base after tumor resection. Pathological examination revealed that the lesion was epidermoid cyst with foreign body granuloma formation (Fig. 2).
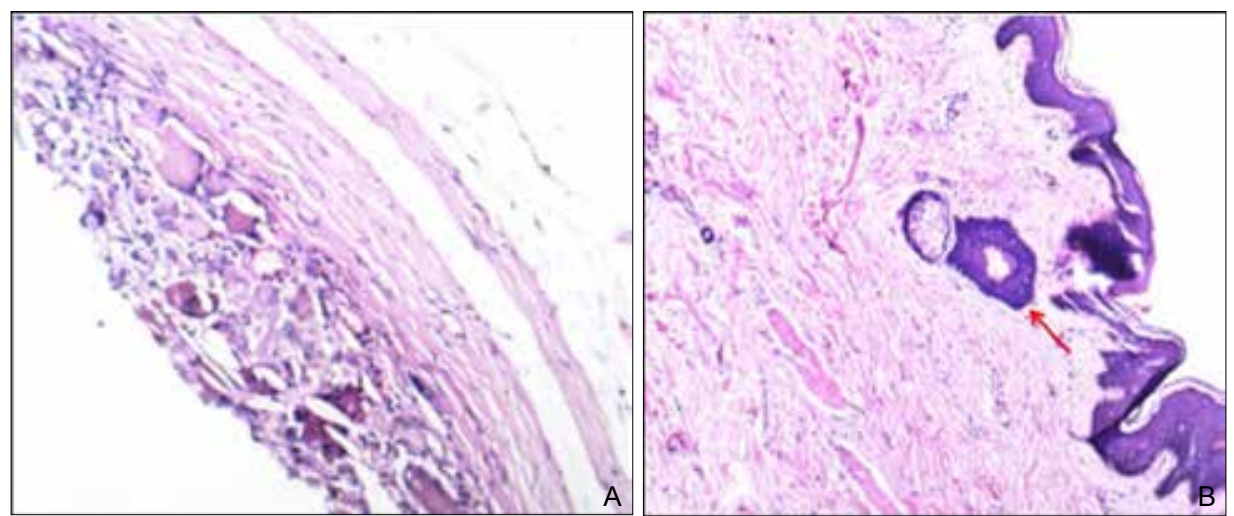

Figure 2 (A) Pathological findings of the specimen shows a number of epidermal cells and foreign body giant cells in surrounding tissues (HE $\times 100)$; (B) The arrow indicates the expanded follicle funnel $(\mathrm{HE} \times 100)$.

\section{Discussion}

The origin of lesions is the key to the judgment of its pathological diagnosis for such skin or subcutaneous soft tissue masses.Sometimes, the differential diagnosis of superficial lesions is very difficult due to the similarity of the imaging appearances and no clear origins. In this case, the ultrasound diagnosis was considered as a dermoid cyst. To retrospectre view the ultrasound imaging, we found that the mass was located in subcutaneous fat layer, and it was very closely related to the skin. Meanwhile, we can found expanded hair follicle structure in deep skin, which implied that the mass was originated from the skin.Pathological inspection also confirmed the expansion of the funnel of the skin follicle. The reason led to make wrong diagnosis in this case was also due to neglecting connection between the mass 
and to the skin and did not see onion-like characteristic changes of epidermoid cysts [4].

Studies conducted by Yasumoto stated [5] that epidermoid cyst featured with thin wall, no interior echo or low echo, no high echo in generally, and the echo was more uniform and clear, while the internal cavity of the dermoid cyst was turbid, mostly showed mixed echoes, and the wall was thick. After carefully review of ultrasound combined with pathological results, we learned something from this case with misdiagnosis. This patient had a long disease history, which developed a non-specific lesion over the time. In general, the epidermoid cyst increases the size over time and the skin repeatedly injured, which causes rupture of the wall. The keratin released during rupture can cause reaction of foreign body granuloma[6], which led to uneven echogenicity within the mass. Thus, the overall lesion could present as mixed hypo- and hyper-echogenic appearance. In summary, skin epidermoid cysts have their own characteristics, which express as a mass originated from the skin, have simple cystic or cystic component with solid changes. The lesion on ultrasound imaging can have the expansive hair follicle-like structure at the skin junction, with onion-like or mixed echogenic changes. Ultrasound imaging can improve diagnostic accuracy through these characteristics along with clinical information.

\section{Conflicts of Interest}

The authors have declared no conflicts of interest. The funders had no role in study design, data collection and analysis, decision to publication, or preparation of the paper.

\section{Reference}

[1] Kim GW, Park JH, Kwon OJ, Kim DH, Kim CW. Clinical Characteristics of Epidermoid Cysts of the External Auditory Canal. J Audiol Otol 2016;20:36-40.

[2] McGavran MH, Binnington B. Keratinous cysts of the skin. Identification and differentiation of pilar cysts from epidermal cysts. Arch Dermatol 1966;94:499-508.

[3] Zheng J, Wang C, Liu F. Intraparenchymal epidermoid cyst: proper surgical management may lead to satisfactory outcome. J Neurooncol 2018;138:591-599.

[4] Atchley JT, Dewbury KC. Ultrasound appearances of testicular epidermoid cysts. Clin Radiol 2000;55:493-502.

[5] Yasumoto M, Shibuya H, Gomi N, Kasuga T. Ultrasonographic appearance of dermoid and epidermoid cysts in the head and neck. $J$ Clin Ultrasound 1991;19:455-461.

[6] Jin W, Ryu KN, Kim GY, Kim HC, Lee JH, Park JS. Sonographic findings of ruptured epidermal inclusion cysts in superficial soft tissue: emphasis on shapes, pericystic changes, and pericystic vascularity. J Ultrasound Med 2008;27:171-176. 